\title{
DEVELOPMENT OF A SENSING SEAT FOR HUMAN AUTHENTICATION
}

\author{
Pioggia G., Ferro M., Zupone G., Chirulli L., De Rossi D. \\ Interdepartmental Research Center "E. Piaggio" - Faculty of Engineering - University of Pisa \\ via Diotisalvi 2, 56100, Pisa, Italy \\ Email: giovanni.pioggia@ing.unipi.it, marcello.ferro@ing.unipi.it
}

Keywords: Sensing seat, unobtrusive piezoresistive sensor array, artificial neural networks, authentication.

\begin{abstract}
In this work the development of a sensing seat for human authentication is reported. Such a system can be used in all the critical scenarios where a seat is available to the human subject. In order to face the authentication task, the sensing seat was developed by means of a novel unobtrusive sensing technology. This is mainly due to two aspects: the unavailability of an existing sensing seat system for human authentication and the inadequacy of the existing sensor technology in order to address the human subject recognition as well as the integration of the sensors in the seat. Thanks to the development of a redundant sensor network, we adopted a hierarchical architecture. Three cooperating classifiers (a distance-based classifier, a KSOM and a MLP) shares the input data and supplying three different classification results. A final classifier (a weighted averager) performs the fusion of the results and supplies the final response.
\end{abstract}

\section{Introduction}

Several companies are currently working in order to realize comfortable interactive seats $[1,2,3,4]$. These systems, some of them already on the market, use different technologies and materials, but they share the use of sensors that measure the pressure exerted on the seat by the passenger. Actually, no existing systems based on sensing seats are currently able to perform the human authentication task and no results on this topic, even if in a preliminary stage, were found. Indeed the sensor technology used in such systems in not adequate to perform the authentication task. That is, information such as the weight of the subject or the level of (dis)comfort may be inferred using a low number of classical sensors. In order to detect and correctly classify the human profile signature, many sensors should be used and the hardware architecture will result in a complex and delicate system. The sensing seat prototype was realised making use of a sensing cover placed between the seat (a truck seat provided by VOLVO) and an external cover that protects the sensors from the external world. The sensing cover is equipped with unobtrusive strain sensors based on Conductive Elastomers (CEs) composites grouped in several patches. CEs composites show piezoresistive properties when a deformation is applied. In order to develop strain sensors, they can be integrated into fabric or other flexible substrates. The sensing cover consists in several arrays of strain sensors directly printed into a Lycra tissue. The resulting design results in a high-impedance circuitry where a reference current is injected. The high-impedance characteristic allows both sensors and wires to be realised by means of the same technology and to gain unobtrusivity. In fact, the use of common electrical wires is avoided within the sensing cover. Moreover the power consumption is quite zero resulting in a completely safe system. A connector plug is placed in one side of the sensing cover in order to connect the system to the front-end module. The fabric with new generation of distributed and redundant smart unobtrusive sensors allows us to guarantee: plasticity, coat whole the seat, low dimension, lightness, directly printed on the fabric, adherence to the seat and low cost. After the study of the signal response on a single strain sensor, several types of topology were taken into account (series, parallel and quadripolar networks) and finally the best compromise between the technical complexity and the signal classification results was found using the series network. A front-end device for the signal conditioning and a data acquisition board were subsequently designed and developed in order to guarantee the flow on data between the sensing system and the PC. The recording protocol was defined as an interactive procedure where the subjects collaborates with the system in order to perform the main tasks (enrollment, authentication). During the enrollment and the authentication steps, the user is asked in turn to seat in some predefined positions. In the case of the enrolment procedure, the new subject is asked to repeat the measurement for 10 times for each predefined position. Then the acquired data are stored into the database and the classifiers are trained again in order to take into account the new user. In the case of the authentication procedure, the user is asked to perform a measurement for each predefined position and the acquired data are compared with the data previously stored into the database. On the basis of the response of the classifiers, the user will be authorised or rejected by the system. The sensing seat system should be adopted in several scenarios where the security is a critical aspect. The system should be available to truck pilots, airplane pilots, plant personnel, people in airports and, in general, people in environments where the security is mandatory and a seat is available to the human subject (truck seat, office seat and airplane seat among the others). 


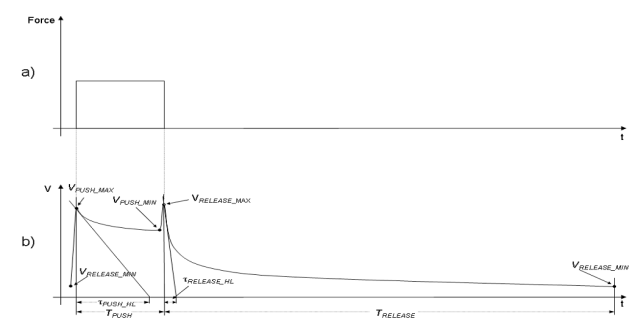

Figure 1: a) A pressure impulse; b) Sensor response a pressure impulse and selected features

\section{Unobtrusive strain sensor array and connections}

The sensing seat was realised by a seat coated by a removable Lycra sensing cover. The sensing cover will be able to respond to simultaneous deformations in different directions by means of a piezoresistive network which consists of a CEs composites rubber screen printed onto a cotton Lycra fabric. The CEs we used are based on a WACKER Ltd (Elastosil LR $3162 \mathrm{~A} / \mathrm{B}$ ) product. It consists in a mixture of graphite and silicon rubber. WACKER Ltd guarantees the non-toxicity of the product. The sensing cover consists in several arrays of strain sensors directly printed into a Lycra tissue. They are elastic and do not modify the mechanical behaviour of the fabric. The resulting design results in a high-impedance circuitry where a reference current is injected. The high-impedance characteristic allows both sensors and wires to be realised by means of the same technology and to gain unobtrusivity. In fact, the use of common electrical wires is avoided within the sensing cover. Moreover the power consumption is quite zero resulting in a completely safe system. A connector plug is placed in one side of the sensing cover in order to connect the system to the front-end module.

Strain impulses result in a typical differential voltage behaviour showed in figure 1. Sensor response shows a peak in correspondence to every mechanical transition. For each signal data are acquired and filtered. Sensor responses during constant pressure time intervals may be approximated by decreasing exponentials, assuming the local minimum as the steady-state value. This approximation results as true as long is the pressure time interval. In order to remove the contribution of high order exponentials, the first order time constants were extracted by means of a window filter. This choice allowed quantization errors introduced by the acquisition device in response to rapid transitions to be avoided and sensor steady state deformation, related to slower frequency components, to be maintained.

Sensor responses during constant pressure time intervals can be approximated by decreasing exponentials, assuming the local minimum as the steady-state value. This approximation results as true as long is the pressure time interval. In order to remove the contribution of high order exponentials, first order time constants were calculated discarding the first $5 \%$ of each curve. This choice allowed quantization errors introduced by the acquisition device in response

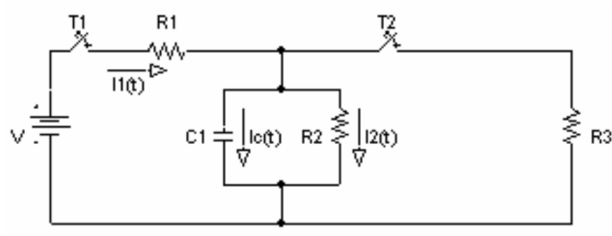

Figure 2: Proposed equivalent electric model of each sensor

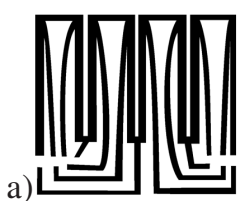

Figure 3: A single sensor array patch to rapid transitions to be avoided and sensor steady state deformation, related to slower frequency components, to be maintained.

In order to model the first-order components of the sensor response (resistance variation) to a rectangular stimulation (applied deformation), the equivalent circuit represented in Fig. 2 can be derived [5].

The power supply $\mathrm{V}$ is the electrical analogous of the imposed deformation. The switch T1 (initially open) is closed and open in correspondence of, respectively, the beginning and the end of the imposed deformation. The switch $\mathrm{T} 2$ (initially open) is closed when T1 is open again. Following a simple analysis of this circuit, it is easy to recognise that the variation of the charging and discharging currents of the circuit in consecutive phases of stimulation are analogous to the variation of the resistance of the sensor during, respectively, its deformation and the following release.

The second step consisted in the design and realization of a two dimensional resistive sensor network in order to redundantly cover the deformable surface of the truck seat. Since a single sensor reading strategy may increase the complexity of the electronic acquisition system endangering the real-time performance, it is possible to adopt several topologies of interconnection of sensors to reduce this complexity. By connecting sensors arrays in electrical networks and reading their borders only, it is possible, in principle, to reconstruct a resistance variation of the value of an inside-located sensor. Nevertheless, these strategies of interconnection lead to a loss in terms of system sensitivity and accuracy in signal reconstruction, hence it is necessary to come to a fair compromise. The strategy we are going to follow is to realise 12 channels single sensor arrays distributed to evenly cover the truck seat. In each sensor array, sensors are serially connected. A current is superimposed in the circuit and high impedance differential voltages are acquired from each sensor. A drawing and a picture of a single sensor array are shown respectively in Fig. 3a and $3 b$. 


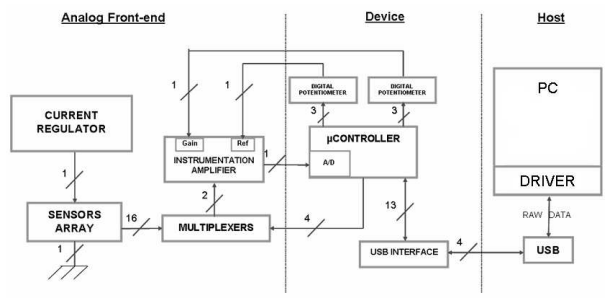

Figure 4: Block schema of the acquisition hardware

a)
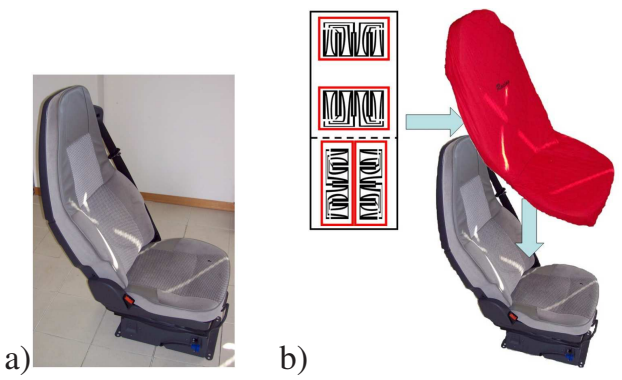

Figure 5: The sensing seat: a) truck seat provided by VOLVO; b) seat cover (the sensors are placed on the back side of the cover)

\section{Hardware design}

The block scheme of the acquisition hardware for a single sensor array is presented in Fig. 4. Two multiplexers allow a sensor to be selected and the relative signal is acquired by a differential amplifier. A microprocessor drives the acquisition block, performs the analogous/digital conversion for all the signals coming from the array and exchanges data packets via USB interface with a scan rate equal to 100 packets/sec. The device is provided with an automatic calibration subsystem which allows gain and offset to be tailored to each sensor.

The data acquisition board (DAQ) consists in a front end card which is able to supply the reference current and to perform a high-impedance measurement for each channel (i.e. each strain sensor). The DAQ communicates with a personal computer using the USB or the PCMCIA protocol (National Instruments 6024E board). A protocol interface is able to abstract in respect to the specific hardware board used for to perform the acquisition. Fig. 5a shows the truck seat provided by VOLVO and Fig. $5 \mathrm{~b}$ the sensing cover.

\section{Architecture of the classifiers}

A software interface is needed in order to perform an initial data filtering and data dispatching to the analysis application [6]. In order to obtain a multitasking architecture, the acquisition task runs in parallel in respect to the analysis task. A message protocol was defined in order to perform the client-server communication. After an initialization step, the server task will communicate new data only when they are available. Currently the server-side HW/SW is able to obtain a sampling rate of 1000 samples per sensor per second. However a sampling rate of 64 samples per sensor per second is sufficient to obtain good results within the sensing seat application.

The analysis task is able to run in real time and to collect new data from the data dispatcher task using a sample-andhold strategy. This task manages the graphical user interface, the network connection to the database and the switching between the operating modalities (enrolment, authentication, monitoring). The software application is developed under the Microsoft Visual C++ 6.0 programming environment. At this time, three classification modules are used during the enrolment and authentication states of the system:

1) A Kohonen Self Organizing Map $[8,9]$ :

- 10x10 integrate-and-fire neurons fully connected to the input vector (36 strain sensors);

- Distance-based learning method;

- Learning rate with decay, learning radius with decay;

- Labelling procedure for the classification task;

- During the test phase, the error level is the Euclidean distance between the input vector and the synaptic weight vector of the winning neuron.

\section{2) A Multi Layer Perceptron [7]:}

- Input layer: 36 integrate-and-fire neurons connected one-to-one to the input vector (36 strain sensors), linear transferring function;

- Hidden layer: 10 integrate-and-fire neurons, fully connected to the input layer and to 1 bias neuron, sigmoid transferring function;

- Output layer: $\mathrm{N}$ integrate-and-fire-neurons, where $\mathrm{N}$ is the number of the enrolled users, fully connected to the hidden layer and to 1 bias neuron;

- Learning rate with decay, momentum with decay;

- Delta-rule learning method;

- During the test phase, the error level is the Euclidean distance between the activation vector of the output layer and the activation vector built on the basis of the logged user information.

3) A distance classifier:

- Distance-based classification method: evaluation of the Euclidean distance between the input vector and the data vector belonging to the logged user;

- The output of this modules is its error level (i.e. the distance described above). 


\subsection{Enrolment State}

During the enrolment state, according to the conceptual design, the data belonging to new user are collected and subsequently stored into the database. The user is also asked to remove wallets and/or coats and to seat in the normal position (with the bottom side and the back side totally adherent to the seat, i.e. the first predefined position). When a steady state is gained for each strain sensor signal, the collected data are stored and the user is asked to seat in the next predefined position (the bottom side is adherent to the seat and the back side is not in contact with the seat, i.e. the second predefined position). When the steady state is reached the collected data are stored and the procedure is repeated for 10 times in order to obtain 10 repeated measurements.

The data set will be rebuild and the SOM and the MLP will be trained again in order to take into account the new user. 500 training epochs are sufficient in order to setup the neural networks. The initial values for the networks parameters are:

\section{1) SOM:}

- Learning rate: 0.9 ;

- Learning rate decay: 0.001;

- Learning radius: 10 ;

- Learning radius decay: 0.001;

2) MLP:

- Learning rate: 0.9;

- Learning rate decay: 0.001;

- Momentum: 0.9;

- Momentum decay: 0.001;

\subsection{Authentication State}

During the authentication state, according to the conceptual design, the data belonging to logged user are collected and the status of the internal classifiers are loaded from the database. The three classifiers will perform the classification task. Each classifier will supply a user identifier and an error level as result of the authentication process.

The authentication output of each sub-module will be taken into account by a final classifier in order to obtain a properly weighted authentication result.

The final output consists in a user identifier and in an identification error level. If the user identifier recognised by the system is not the same of the login information, the user will be rejected. If the user identifier recognised by the sensing seat system is equal to the login information identifier and the error level is less than a predefined threshold, then the user will be authorised by the sensing seat system.

\subsection{Limits of the existing architecture}

The sensing seat system is able to operate a classification on the basis of the deformation of the sensing cover caused by the human subject when seated on the sensing seat in different predefined positions. Three main problems may occur, making the system unable to correctly perform the classification task:

1. presence of wallet and/or coat

2. changes in the way the same human subject seats in each predefined position

3. changes in the human subject's profile (aging, changes in weight)

For each of these situations, the final prototype system will able to try to solve the problem. The point 1) will result in the human subject signature to be not so 'far' from the signature information stored into the database. Both the enrolment and the authentication procedures are driven by the system, that will inform the user to remove wallets and coat before than starting the session. However, since each classifier of the sensing seat system is able to supply an error level, a threshold should be defined in order to detect if the system does not know if the user has to be rejected. I this is the case, a system waring should be given through the GUI and the user should be asked again to remove wallets and coat.

The points 2) and 3) are difficult to be taken into account. However the time-stamp information for each measurement in the signature was taken into account for this kind of situations. We will suppose that the changes (the way of sitting, the weight, the aging) will be small especially if they are considered in short period of time. Once the user is enrolled the time-stamp is saved into the signature for each measurement (related to several repeated measurement for each predefined position). If the user continuously performs the authentication task in the system (let us say, once a month at least) and if he will be correctly authenticated, the new measurements (probably a bit different from the previous measurement) should be saved into the database and the classifiers should be trained again taking into account a LIFO queue of measurements

\section{Preliminary Results}

Tests on 10 human subjects were used in the preliminary measurement campaign. 10 repeated measures were carried out for each human subject. The authentication results (through the evaluation of the confusion matrix) assesses as follows:

- An enrolled subject claims to be himself: $93 \%+/-2 \%$ correct recognition

- A unenrolled subject claims to be an enrolled subject (intrusion): $97 \%+/-2 \%$ correct rejection. 


\section{Conclusions}

In this paper the development of a novel sensing seat based on unobtrusive piezoresistive sensor array is described. The main result is a positive assessment of the use of the reported sensing seat in the authentication task. Based on preliminary results, the former objective should be at the hand. Another relevant result is the assessment of the sensor technology and the classification software.

\section{Acknoledgements}

This work was supported by the HUMABIO European Project, contract n. 026990.

\section{References}

[1] http://www.tekscan.com/industrial/app_seating.html

[2] http://www.novel.de/

[3] http://www.softswitch.co.uk/

[4] http://www.johnsoncontrols.com/comfortlab/

[5] Pioggia G., Ferro M., De Rossi D., Lorussi F., Carpi F., Labbozzetta E., Di Francesco F., A biomimetic sensing skin: characterization of piezoresistive fabric-based elastomeric sensors, in Sensors and Microsystems, 10th Italian Conference, World Scientific Publishing Co., Singapore, 2005.

[6] Pioggia G., Ferro M., Di Francesco F., Dalle Mura G., De Rossi D., An Architecture for High Efficiency Realtime Sensor and Actuator Data Processing, EUROSENSORS XIX, Barcelona, Spain, September 11th14th, 2005.

[7] Kinnebrock, W., 1992. Neural Networks, Munchen, Oldenburg Verlag.

[8] Kohonen, T., 1988. Self-Organization and Associative Memory, Springer, second edition.

[9] Kohonen, T., 1997. Self-Organising Maps, Springer Series in Information Sciences. Vol. 30. Springer, Berlin, Heidelberg, New York, 2nd extended edition. 